\title{
EXACT SAMPLING RESULTS FOR SIGNALS WITH FINITE RATE OF INNOVATION USING STRANG-FIX CONDITIONS AND LOCAL KERNELS
}

\author{
Pier Luigi Dragotti
}

\author{
Electrical and Electronic Engineering \\ Imperial College London \\ Exhibition Road, London SW7-2AZ, UK \\ p.dragotti@imperial.ac.uk
}

\author{
Martin Vetterli ${ }^{1}$ and Thierry Blu ${ }^{2}$ \\ ${ }^{1} \mathrm{LCAV},{ }^{2} \mathrm{BIG}$ \\ École Polytechnique Fédérale de Lausanne \\ CH - 1015 Lausanne, Switzerland. \\ \{martin.vetterli,thierry.blu\}@epfl.ch
}

\begin{abstract}
Recently, it was shown that it is possible to sample classes of signals with finite rate of innovation [7]. These sampling schemes, however, use kernels with infinite support and this leads to complex and instable reconstruction algorithms.

In this paper, we show that many signals with finite rate of innovation can be sampled and perfectly reconstructed using kernels of compact support and a local reconstruction algorithm. The class of kernels that we can use is very rich and includes any function satisfying Strang-Fix conditions, Exponential Splines and functions with rational Fourier transforms.
\end{abstract}

\section{INTRODUCTION}

Sampling theory plays a central role in modern signal processing and communications, and has experienced a recent revival thanks, in part, to the recent advances in wavelet theory [5, 4]. In the typical sampling setup, the original continuous-time signal $x(t)$ is filtered before being (uniformly) sampled with sampling period $T$. If we call $y(t)=h(t) * x(t)$ the filtered version of $x(t)$, then the samples $y_{n}$ are given by $y_{n}=\langle x(t), \varphi(t / T-n)\rangle$ where the sampling kernel $\varphi(t)$ is the time-reversed version of $h(t)$.

Recently, it was shown that it is possible to develop sampling schemes for classes of signals that are neither bandlimited nor belong to a fixed sub-space [7]. For instance, it was shown that it is possible to sample streams of Diracs or piecewise polynomial signals using a sinc or a Gaussian kernel. The common feature of such signals is that they have a parametric representation with a finite number of degrees of freedom and are, therefore, called signals with finite rate of innovation (FRI) [7]. The reconstruction process is based on the use of a locator or annihilating filter, a tool widely used in spectral estimation [2] and error correction coding [1].

The fundamental limit of the above method, as well as of the classical Shannon reconstruction scheme, is that they use kernels of infinite support. As a consequence, the reconstruction algorithm is usually physically non-realizable (e.g., realization of an ideal low-pass filter) or, in the case of FRI signals, becomes immediately complex and instable (the complexity is in fact influenced by the global rate of innovation of $x(t)$ ).

In this paper we show that many signals with a local finite rate of innovation can be sampled and perfectly reconstructed using a wide range of sampling kernels and a local reconstruction algorithm. In particular, we show that the main property the kernel has to satisfy is to be of compact support and able to reproduce polynomials or exponentials. More precisely, if $\varphi(t)$ is the kernel, we need it to satisfy

$$
\sum_{n} c_{m, n} \varphi(t-n)=t^{m} \quad m=0,1, \ldots, N
$$

or

$\sum_{n} c_{m, n} \varphi(t-n)=e^{a_{m} t} \quad m=0,1, \ldots, N$, and $a_{m}=\alpha_{0}+m \lambda$

for a proper choice of the coefficients $c_{m, n}$. Interesting enough, the reconstruction algorithm proposed in this paper is also based on the annihilating filter method. We also show, with a simple example, that in some cases it is possible to relax the hypothesis of compactly supported kernels and that, for instance, many kernels with rational transfer functions can be used to sample FRI signals. Despite the fact that these kernels have infinite support, the reconstruction algorithm remains local and its complexity still depends on the local, rather than global, rate of innovation of $x(t)$.

The paper is organized as follows: in the next section, we briefly review the notion of FRI signals and the annihilating filter method. Section 3 presents new sampling theorems for classes of FRI signals using kernels that can reproduce polynomials. In Section 4, we review the notion of Exponential Splines [6] and extend our sampling schemes to the case of kernels that can reproduce exponentials. Further extensions are discussed in Section 5 where we also show how to estimate FRI signals at the output of an $R C$ circuit. We conclude in Section 6.

\section{PRELIMINARIES}

In this section, we give a brief review of signals with finite rate of innovation and of the annihilating filter method. The notion of FRI signals was introduced in [7]. For a more detailed treatment of the annihilating filter method, we refer to [2].

\subsection{Signals with Finite Rate of Innovation}

Consider a signal of the form

$$
x(t)=\sum_{n \in \mathbb{Z}} \sum_{k=0}^{K} \lambda_{n, k} \varphi_{k}\left(\frac{t-t_{n}}{T}\right) .
$$

Clearly, if the set of functions $\left\{\varphi_{k}(t)\right\}_{k=0,1, \ldots, K}$ is known, the only free parameters in the signal $x(t)$ are the coefficients $\lambda_{n, k}$ and 
the time shifts $t_{n}$. It is therefore natural to introduce a counting function $C_{x}\left(t_{a}, t_{b}\right)$ that counts the number of free parameters in $x(t)$ over an interval $\left[t_{a}, t_{b}\right]$. The rate of innovation of $x(t)$ is then defined as [7]

$$
\rho=\lim _{\tau \rightarrow \infty} \frac{1}{\tau} C_{x}\left(-\frac{\tau}{2}, \frac{\tau}{2}\right) .
$$

Definition 1 ([7]) A signal with a finite rate of innovation is a signal whose parametric representation is given in (3) and with a finite $\rho$ as defined in (4).

It is of interest to note that shift-invariant signals, including bandlimited signals, are included in Definition 3. For instance, if we call $f_{\max }$ the maximum non-zero frequency in a bandlimited real signal, then $\rho=2 f_{\max }$.

In some cases it is more convenient to consider a local rate of innovation with respect to a moving window of size $\tau$. The local rate of innovation at time $t$ is thus given by [7]

$$
\rho_{\tau}(t)=\frac{1}{\tau} C_{x}\left(t-\frac{\tau}{2}, t+\frac{\tau}{2}\right) .
$$

Clearly $\rho_{\tau}(t)$ tends to $\rho$ as $\tau \rightarrow \infty$.

\subsection{The annihilating filter method}

Assume that we observe a signal

$$
s[m]=\sum_{k=0}^{K-1} a_{k} u_{k}^{m} \quad m \in \mathbb{Z}
$$

and that we want to find the weights $a_{k}$ and the locations $u_{k}$ of $s[m]$. Call $h[m] m=0,1, \ldots, K$ a filter with $z$-transform

$$
H(z)=\sum_{m=0}^{K} h[m] z^{-m}=\prod_{k=0}^{K-1}\left(1-u_{k} z^{-1}\right) .
$$

That is, the roots of $H(z)$ correspond to the locations $u_{k}$. It clearly follows that

$$
h[m] * s[m]=\sum_{i=0}^{K} h[i] s[m-i]=0 .
$$

The filter $h[m]$ is thus called annihilating filter since it annihilates the observed signal $s[m]$. Notice that $h[m]$ is unique for the observed signal since the locations $u_{k}$ are distinct.

The knowledge of $h[m]$ is sufficient to retrieve the locations $u_{0}, u_{1}, \ldots, u_{K-1}$, since these locations are the roots of the polynomial in (7). The filter coefficients $h[m]$ must be such that (8) is satisfied. Since there are $K$ unknown coefficients (recall that $h[0]=1$ ), we need at least $K$ linear equations. The identity in (8) leads to a Yule-Walker system of equations involving $2 K$ consecutive values of $s[m]$ and, in this case, it has a unique solution since $h[m]$ is unique for the given signal. Given the filter coefficients $h[m]$ and therefore the locations $u_{0}, u_{1}, \ldots, u_{k}$; the weights $a_{k}$ are obtained by solving $K$ consecutive equations in (6). These equations form a Vandermonde system which yields a unique solution for the weights $a_{k}$ given that the $u_{k}$ s are distinct.

In the following sections, we will show that our sampling problem can always be reduced to that of finding the weights and the shifts of a signal like $s[m]$.

\section{RECONSTRUCTION OF FRI SIGNALS USING LOCAL KERNELS THAT REPRODUCE POLYNOMIALS}

We assume that the sampling kernel $\varphi(t)$ is of compact support $L$, that is, $\varphi(t) \neq 0$ for $t \in[-L / 2, L / 2]$ and that $\varphi(t)$ satisfies the Strang-Fix conditions [3], that is, a linear combination of shifted versions of $\varphi(t)$ can reproduce polynomials of maximum degree $N$ (see Equation (1)). We consider the case of streams of Diracs first and derive the other results directly from this case.

\subsection{Streams of Diracs}

Theorem 1 Given is a sampling kernel $\varphi(t)$ that can reproduce polynomials of maximum degree $N \geq 2 K-1$ and of compact support $L$. An infinite-length stream of Diracs $x(t)=\sum_{n \in \mathbb{Z}} a_{n} \delta(t-$ $\left.t_{n}\right)$ is uniquely determined from the samples defined by $y_{n}=$ $\langle\varphi(t / T-n), x(t)\rangle$ if and only if there are at most $K$ Diracs in an interval of length $K L T$.

Proof: Assume for simplicity that $T=1$ and assume for now that $x(t)$ contains only $K$ Diracs, that is, $x(t)=\sum_{k=0}^{K-1} a_{k} \delta(t-$ $\left.t_{k}\right)$ and $t \in \mathbb{R}$. Call $s[m]=\sum_{n} c_{m, n} y_{n}, m=0,1, \ldots, N$ the weighted sum of the observed (non-zero) samples, where the weights $c_{m, n}$ are those in Equation (1). We have that

$$
\begin{aligned}
s[m] & =\sum_{n} c_{m, n} y_{n} \\
& \stackrel{(a)}{=}\left\langle x(t), \sum_{n} c_{m, n} \varphi(t-n)\right\rangle \\
& \stackrel{(b)}{=} \quad \int_{-\infty}^{\infty} x(t) t^{m} d t \\
& \stackrel{(c)}{=} \quad \sum_{k=0}^{K-1} a_{k} t_{k}^{m} \quad m=0,1, \ldots, N
\end{aligned}
$$

where (a) follows from the linearity of the inner product, (b) from the polynomial reproduction formula in (1), and (c) from the fact that $x(t)=\sum_{k=0}^{K-1} a_{k} \delta\left(t-t_{k}\right)$. Hence by opportunely combining the samples $y_{n}$, we end-up observing a signal $s[m]$ of the form $s[m]=\sum_{k=0}^{K-1} a_{k} t_{k}^{m}, m=0,1, \ldots, N$ which is equivalent to the signal in (6). It is thus possible to retrieve the amplitudes $a_{k}$ and the locations $t_{k}$ of the Diracs using the annihilating filter method.

Call $h[m] m=0,1, \ldots, K$ the filter that annihilates $s[m]$. This filter is unique since the locations of the Diracs are distinct and we are assuming $h[0]=1$. We observe $s[0], s[1], \ldots, s[N]$, therefore the $K$ unknown coefficients of $h[m]$ are the solution of the Yule-Walker system produced by the identity in (8). Notice that, since we need at least $2 K$ values of $s[m]$, it is crucial that the sampling kernel $\varphi(t)$ be able to reproduce polynomials of degree $N \geq 2 K-1$. Once the filter coefficients are found, the Diracs' locations are the roots of the polynomial $p(z)=\sum_{k=0}^{K} h[m] z^{-m}$. Finally the amplitudes of the Diracs are obtained by solving the first $K$ equations in (9). These equations form a Vandermonde system which has a unique solution since the $t_{k} \mathrm{~s}$ are distinct. Hence, the annihilating filter methods allows us to retrieve the original signal from its samples.

In general, $x(t)$ may contains more than $K$ Diracs. However, since the kernel is of compact support, only a finite number of samples is influenced by a certain set of Diracs. Thus, if we are sure that the samples generated by different sets of $K$ Diracs do not influence each other, we can still use the above method sequentially. It is easy to see that this happens when there are no more the $K$ 
Diracs in an interval of size $K L T$ or, using the terminology introduced in the previous section, when the local rate of innovation $\rho_{K L T}(t) \leq 2 / L T$.

Notice that the complexity of the algorithm does not depend on the total number of Diracs present in the signal, but only on the maximum number of Diracs that we can have in a certain interval. That is the complexity of the algorithm depends only on the local rate of innovation of $x(t)$.

We conclude this section be highlighting that, with an approach similar to the one presented above, one can sample streams of differentiated Diracs as well. However, we omit the proof due to lack of space.

Theorem 2 Given is a sampling kernel $\varphi(t)$ that can reproduce polynomials of maximum degree $N \geq 2 K M-1$ and of compact support L. An infinite-length stream of differentiated Diracs $x(t)=\sum_{n=\mathbb{Z}} \sum_{m=0}^{M-1} a_{n, m} \delta^{(m)}\left(t-t_{n}\right)$ is uniquely determined by the samples $y_{n}=\langle\varphi(t / T-n), x(t)\rangle$ if and only if there are at most $K$ differentiated Diracs in an interval of length $K L T$.

\subsection{Piecewise polynomial signals}

A signal $x(t)$ is piecewise polynomial with pieces of maximum degree $M$ if and only if its $(M+1)$ derivative is a stream of differentiated Diracs or $x(t)^{(M+1)}(t)=\sum_{n \in \mathbb{Z}} \sum_{m=0}^{M} a_{n, m} \delta^{(m)}\left(t-t_{n}\right)$. This means that if we are able to relate the samples of $x(t)$ to those of $x^{(M+1)}(t)$, we can use Theorem 2 to reconstruct $x(t)$. This is indeed possible by recalling the link existing between discrete differentiation and derivation in continuous domain.

Consider the samples $y_{n}=\langle x(t), \varphi(t-n)\rangle$ where $\varphi(t)$ is a generic sampling kernel. Let $z_{n}^{(1)}$ denote the finite difference $y_{n+1}-y_{n}$. It follows that

$$
\begin{aligned}
z_{n}^{(1)} & =\langle x(t), \varphi(t-n-1)-\varphi(t-n)\rangle \\
& =\frac{1}{2 \pi}\left\langle X(\omega), \hat{\varphi}(\omega) e^{-j \omega n}\left(e^{-j \omega}-1\right)\right\rangle \\
& =\frac{1}{2 \pi}\left\langle X(\omega),-j \omega \hat{\varphi}(\omega) e^{-j \omega n}\left(\frac{1-e^{-j \omega}}{j \omega}\right)\right\rangle \\
& =\left\langle\frac{d x(t)}{d t}, \varphi(t-n) * \beta_{0}(t-n)\right\rangle
\end{aligned}
$$

where $\beta_{0}(t)$ is the B-Spline of order zero. This means that the coefficients $z_{n}^{(1)}$ represent the samples given by the inner products of the derivative of $x(t)$ with the new kernel $\varphi(t) * \beta_{0}(t)$. In the same way, it is straight-forward to show that the $(M+1)$ th finite differences $z_{n}^{(M+1)}$ represent the samples obtained by sampling $x^{(M+1)}(t)$ with the kernel $\varphi(t) * \beta_{M}(t)$, where $\beta_{M}(t)$ is the BSpline of degree $M$.

Now, assume that $\varphi(t)$ is of compact support $L$ and that it can reproduce polynomials of maximum degree $N$. Then $\varphi(t) *$ $\beta_{M}(t)$ has support $L+M+1$ and can reproduce polynomials of maximum degree $N+M+1$. Thus, if the new kernel satisfies the hypotheses of Theorem 2, the samples $z_{n}^{(M+1)}$ are a sufficient representation of $x^{(M+1)}(t)$ and, therefore, of $x(t)$. This leads to the following theorem

Theorem 3 Given is a sampling kernel $\varphi(t)$ of compact support $L$ and that can reproduce polynomials of maximum degree $N$. An infinite-length piecewise polynomial signal with pieces of maximum degree $M-1$ is uniquely defined by the samples $y_{n}=$ $\langle\varphi(t / T-n), x(t)\rangle$ if and only if there are at most $K+1$ polynomials in an interval of size $(L+M) K T$ and $2 K M-1 \leq(M+N)$.

Proof: Assume again $T=1$. Given the samples $y_{n}$, compute the $M$ th finite difference $z_{n}^{(M)}$. As shown before, $z_{n}^{(M)}=\left\langle x^{(M)}(t), \varphi(t-\right.$ $\left.n) * \beta_{M-1}(t-n)\right\rangle$ and $x(t)^{(M)}(t)=\sum_{n \in \mathbb{Z}} \sum_{m=0}^{M-1} a_{n, m} \delta^{(m)}(t-$ $\left.t_{n}\right)$. The new kernel $\varphi(t) * \beta_{M-1}(t)$ has support $L+M$ and can reproduce polynomials of maximum degree $N+M$. Since for hypothesis $x(t)$ has at most $K+1$ polynomial in an interval of size $(L+M) K, x^{(M)}(t)$ has at most $K$ Diracs in that interval with a total number of weights $\hat{K}=K M$. Since we are assuming $2 K M-1 \leq N+M$, the hypotheses of Theorem 2 are satisfied, thus, the samples $z_{n}^{(M)}$ are sufficient to reconstruct $x^{(M)}(t)$ and therefore $x(t) .{ }^{1}$

\section{THE EXPONENTIAL CASE}

In the previous section we have used the property that $\varphi(t)$ can reproduce polynomials to reduce our sampling problem to that of finding the coefficients $a_{k}$ and $t_{k}$ of the discrete signal $s[m]=$ $\sum_{k=0}^{K-1} a_{k} t_{k}^{m}$. Then these coefficients can be determined using a classical annihilating filter method. The annihilating filter method, however, can be used also if $s[m]$ is given by $s[m]=\sum_{k=0}^{K-1} a_{k} e^{\lambda m t}$ This means that we can sample FRI signals also if the kernel is able to reproduce exponentials.

The theory related to the reproduction of exponentials is somewhat more recent and relies on the notion of Exponential Splines (E-Splines) [6]. A function $\beta_{\alpha}(t)$ with Fourier transform $\hat{\beta}_{\alpha}(\omega)=$ $\frac{1-e^{\alpha-j \omega}}{j \omega-\alpha}$ is called E-Spline of first order. Notice that $\alpha$ does not have to be real, but can be any complex number. Moreover, notice that $\beta_{\alpha}(t)$ reduces to the classical zero-order B-Spline when $\alpha=0$. The function $\beta_{\alpha}(t)$ satisfies several interesting properties, in particular, it is of compact support and a linear combination of shifted versions of $\beta_{\alpha}(t)$ reproduces $e^{\alpha t}$. As in the classical case, higher order E-Splines are obtained by successive convolutions of lower-order ones or $\hat{\beta}_{\vec{\alpha}}(\omega)=\prod_{n=0}^{N} \frac{1-e^{\alpha_{n}-j \omega}}{j \omega-\alpha_{n}}$ where $\vec{\alpha}=\left(\alpha_{0}, \alpha_{1}, \ldots, \alpha_{N}\right)$. The higher-order Spline is again of compact support and it is possible to show that it can reproduce any exponential in the subspace spanned by $\left\{e^{a_{0} t}, e^{a_{1} t}, \ldots, e^{a_{N} t}\right\}[6]$.

Now assume that our kernel is of compact support and that it is able to reproduce exponential of the form $e^{\alpha_{m} t}$ with $\alpha_{m}=$ $\alpha_{0}+m \lambda$ and $m=0,1, \ldots, N$. For instance, $\varphi(t)$ might be an E-Spline $\beta_{\vec{\alpha}}(t)$ with $\vec{\alpha}=\left(\alpha_{0}, \alpha_{1}, \ldots, \alpha_{N}\right)$ and $\alpha_{m}=\alpha_{0}+m \lambda$ or a composite function $\varphi(t) * \beta_{\vec{\alpha}}(t)$ [6]. Consider again a stream of $K$ Diracs $x(t)=\sum_{k=0}^{K-1} a_{k} \delta\left(t-t_{k}\right)$ The samples $y_{n}$ are then given by $y_{n}=\langle x(t), \varphi(t-n)\rangle$ and, using Eq. (2), it follows that

$$
\begin{aligned}
s[m] & =\sum_{n} c_{m, n} y_{n}=\int_{-\infty}^{\infty} x(t) e^{\alpha_{0}+m \lambda t} d t \\
& =\sum_{k=0}^{K-1} a_{k} e^{\alpha_{0}+m \lambda t_{k}} \quad m=0,1, \ldots, N .
\end{aligned}
$$

This means that, as in the polynomial case, by opportunely combining the samples $y_{n}$ we end-up observing a signal $s[m]$ of the form $s[m]=\sum_{k=0}^{K-1} a_{k} e^{\alpha_{0}+m \lambda t_{k}}$. We can therefore reconstruct $x(t)$ using the annihilating filter method of Section 2. Again, this reconstruction algorithm can be applied to any stream of Diracs with local rate of innovation $\rho_{K L T}(t) \leq 2 / L T$. Thus we have

\footnotetext{
${ }^{1}$ Note that the mean of $x(t)$ is obtained directly.
} 
Theorem 4 Given is a sampling kernel $\varphi(t)$ of compact support $L$ and that can reproduce exponentials $e^{\alpha_{0}+m \lambda t}$ with $m=0,1, \ldots, N$ and $N \geq 2 K-1$. An infinite-length stream of Diracs $x(t)=$ $\sum_{n \in \mathbb{Z}} a_{n} \delta\left(t-t_{n}\right)$ is uniquely determined from the samples defined by $y_{n}=\langle x(t), \varphi(t / T-n)\rangle$ if and only if there are at most $K$ Diracs in an interval of length KLT.

\section{RECONSTRUCTION OF FRI SIGNALS AT THE OUTPUT OF AN ELECTRIC CIRCUIT}

We conclude this paper by showing how to estimate a piecewise constant signal at the output of an electrical circuit. This reconstruction algorithm relies on the results of the previous sections, but also on the fact that it is possible to convert any kernel with Fourier transform $\hat{\varphi}_{\vec{\alpha}}(\omega)=\frac{\hat{\varphi}(\omega)}{\prod_{n=0}^{N} j \omega-\alpha_{n}}$ into the composite kernel $\varphi(t) * \beta_{\vec{\alpha}}(t)$. This is done by filtering the samples $y_{n}$ with an FIR filter $h[n]$ with $z$-transform $H(z)=\prod_{n=0}^{N}\left(1-e^{\alpha_{n}} z^{-1}\right)$.

Consider the classical $R C$ circuit shown in Figure 1 and call $H(\omega)=\alpha /(\alpha+j \omega)$ with $\alpha=1 / R C$ its transfer function. Assume that the input voltage is $x(t)=A u\left(t-t_{0}\right)$. The output $y(t)=h(t) * x(t)$ is clearly given by $y(t)=A u\left(t-t_{0}\right)-$ $A e^{-\alpha\left(t-t_{0}\right)} u\left(t-t_{0}\right)$. The output voltage is then uniformly sampled with sampling period $T=1$ leading to the samples $y_{n}=$ $A u\left(n-t_{0}\right)-A e^{-\alpha\left(n-t_{0}\right)} u\left(n-t_{0}\right)$. Alternatively, we can say that $y_{n}=\langle x(t), \varphi(t-n)\rangle$ with $\varphi(t)=h(-t) .{ }^{2}$ Our aim is to retrieve $x(t)$ from the samples $y_{n}$.

First, compute the following difference

$$
\begin{aligned}
z_{n} & =e^{\alpha} y_{n+1}-y_{n}=\left\langle x(t), e^{\alpha} \varphi(t-n-1)-\varphi(t-n)\right\rangle \\
& =\frac{1}{2 \pi}\left\langle X(\omega), \alpha e^{-j \omega n} \frac{\left(1-e^{\alpha-j \omega}\right)}{(j \omega-\alpha)}=\left\langle x(t), \alpha \beta_{\alpha}(t-n)\right\rangle .\right.
\end{aligned}
$$

Then compute the first order difference

$$
z_{n}^{(1)}=z_{n+1}-z_{n}=\left\langle\frac{d x(t)}{d t}, \alpha \beta_{\alpha}(t-n) * \beta_{0}(t-n)\right\rangle .
$$

Now, the signal $d x(t) / d t$ is a Dirac centered at $t_{0}$ and with amplitude $A$. The new kernel $\varphi_{\alpha}(t)=\alpha \beta_{\alpha}(t) * \beta_{0}(t)$ is of compact support $L=2$, and can reproduce a constant function or the exponential $e^{\alpha t}$. More precisely

$$
\frac{1}{e^{\alpha}-1} \sum_{n} \varphi_{\alpha}(t-n)=1
$$

and

$$
\frac{1}{1-e^{-\alpha}} \sum_{n} e^{\alpha n} \varphi_{\alpha}(t-n)=e^{\alpha t}
$$

This means that (see Theorem 4) $\frac{1}{e^{\alpha}-1} \sum_{n} z_{n}^{(1)}=A$ and that $\frac{1}{1-e^{-\alpha}} \sum_{n} e^{\alpha n} z_{n}^{(1)}=A e^{\alpha t_{0}}$. Thus, we retrieve the amplitude $A$ from the first sum and the location $t_{0}$ from the second one.

Let us verify the above analysis for our specific example. Recall that in our case $y_{n}=A u\left(n-t_{0}\right)-A e^{-\alpha\left(n-t_{0}\right)} u\left(n-t_{0}\right)$ and assume for simplicity that $t_{0} \in[0,1]$, then

$$
z_{n}=e^{\alpha} y_{n+1}-y_{n}= \begin{cases}0 & \text { for } n<0 \\ A\left(e^{\alpha}-e^{\alpha t_{0}}\right) & \text { for } n=0 \\ A\left(e^{\alpha}-1\right) & \text { for } n>0\end{cases}
$$

\footnotetext{
${ }^{2}$ Recall that, since $h(t)$ is real, $\varphi(t)=h(-t)$ implies that $\hat{\varphi}(\omega)=$ $H(-\omega)$.
}

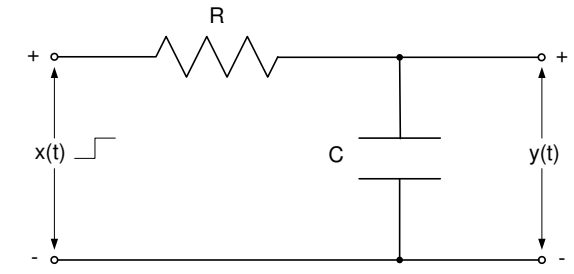

Fig. 1. A typical $R C$ circuit. In our example the input voltage is $x(t)=A u\left(t-t_{0}\right)$ and the output $y(t)$ is uniformly sampled. The samples $y_{n}$ are sufficient to reconstruct $x(t)$ exactly.

and

$$
z_{n}^{(1)}=z_{n+1}-z_{n}= \begin{cases}A\left(e^{\alpha}-e^{\alpha t_{0}}\right) & \text { for } n=-1 \\ A\left(e^{\alpha t_{0}}-1\right) & \text { for } n=0 \\ 0 & \text { otherwise }\end{cases}
$$

Therefore, it is clearly true that $1 /\left(e^{\alpha}-1\right) \sum_{n} z_{n}^{(1)}=A$ and that $1 /\left(1-e^{-\alpha}\right) \sum_{n} e^{\alpha n} z_{n}^{(1)}=A e^{\alpha t_{0}}$.

Notice that with this $R C$ circuit we can sample any piecewise constant signal that has at most one discontinuity in an interval of length $2 T$. To sample signals with higher local rate of innovation, we need an electrical circuit with more than one pole. For instance, we can use a properly designed cascade of $R C$ circuits.

\section{CONCLUSIONS}

In this paper we have shown that it is possible to sample FRI signals with kernels that can reproduce polynomials or exponentials and with a local reconstruction algorithm. Applications of these sampling theorems can potentially be found in signal processing, communication systems and biological systems.

\section{REFERENCES}

[1] R.E. Blahut. Theory and Practice of Error Control Codes. Addison-Wesley, 1983.

[2] P. Stoica and R Moses. Introduction to Spectral Analysis. Englewood Cliffs,NJ, Prentice-Hall, 2000.

[3] G. Strang and Fix. G. A Fourier analysis of the finite element variational method. In Constructive Aspect of Functional Analysis, pages 796-830, Rome, Italy, 1971.

[4] M. Unser. Sampling-50 years after Shannon. Proc. IEEE, 88:569-587, April 2000.

[5] M. Unser and A. Aldroubi. A general sampling theory for nonideal acquisition devices. IEEE Trans. Signal Processing, 42(11):2915-2925, November 1994.

[6] M. Unser and T. Blu. Cardinal Exponential Splines: Part ITheory and Filtering Algorithms. IEEE Trans. on Signal Processing, to appear, 2004.

[7] M. Vetterli, P. Marziliano, and T. Blu. Sampling signals with finite rate of innovation. IEEE Trans. Signal Processing, 50(6):1417-1428, June 2002. 